\title{
Awareness of knowledge or awareness of processing? Implications for sleep-related memory consolidation
}

\author{
Juliana Yordanova ${ }^{1,2}$, , Vasil Kolev ${ }^{1,2}$ and Rolf Verleger ${ }^{1}$ \\ Department of Neurology, University of Lübeck, Lübeck, Germany \\ 2 Institute of Neurobiology, Bulgarian Academy of Sciences, Sofia, Bulgaria
}

\section{Edited by:}

Francisco Barceló, University of llles

Balears, Spain

\section{Reviewed by:}

Mercedes Atienza, University Pablo de

Olavide, Spain

Sara C. Mednick, University of

California, USA

\section{*Correspondence}

Juliana Yordanova, Department of Cognitive Psychophysiology, Institute of Neurobiology, Bulgarian Academy of Sciences, Acad. G. Bonchev. Str., Block 23, 1113 Sofia, Bulgaria.

e-mail: jyord@bio.bas.bg
The present study assessed the effects of awareness at encoding on off-line learning during sleep. A new framework is suggested according to which two aspects of awareness are distinguished: awareness of task information, and awareness of task processing. The number reduction task (NRT) was employed because it has two levels of organization, an overt one based on explicit knowledge of task instructions, and a covert one based on hidden abstract regularities of task structure (implicit knowledge). Each level can be processed consciously (explicitly) or non-consciously (implicitly). Different performance parameters were defined to evaluate changes between two sessions for each of the four conditions of awareness arising from whether explicit or implicit task information was processed explicitly or implicitly. In two groups of subjects, the interval between the pre-sleep and post-sleep sessions was filled either with early-night sleep, rich in slow wave sleep (SWS), or late-night sleep, rich in rapid eye movement (REM) sleep. Results show that implicit processing of explicit information was improved in the post-sleep relative to the pre-sleep session only in the early-night group. Independently of sleep stage, changes between sessions occurred for explicit processing of implicit information only in those subjects who gained insight into the task regularity after sleep. It is concluded that SWS but not REM sleep specifically supports gains in computational skills for the processing of information that was accessible by consciousness before sleep.

Keywords: memory consolidation, sleep, implicit knowledge, explicit knowledge, implicit processing, explicit processing, insight

\section{INTRODUCTION}

There is now increasing evidence that sleep supports memory consolidation (Karni et al., 1994; Maquet, 2001; Walker et al., 2002; Walker and Stickgold, 2004; Born et al., 2006). Consolidation refers to off-line processes leading to enhancement or stabilization of memory representations in the absence of any practice (on-line processing), as demonstrated by performance improvement and resistance to interference after retention intervals. In contrast to practice-based learning during wake, off-line learning can take place during both wake and sleep (Robertson et al., 2004; Cohen et al., 2005; Krakauer and Shadmehr, 2006). Yet, neural networks supporting off-line learning during wake are different from those supporting off-line learning during sleep (Robertson et al., 2005; Peigneux et al., 2006; Diekelmann and Born, 2007; Kirov et al., 2009). Sleep-dependent consolidation is proposed to engage hippocampal-neocortical circuits (McClelland et al., 1995), in contrast to wake-dependent consolidation (Robertson, 2009). The hippocampal-neocortical model (McClelland et al., 1995) assumes that the new information encoded during wake is stored temporarily in an intermediate buffer including the medial temporal lobe and the hippocampus. During sleep this information is transferred to a long-term store in the neocortex. The meaning of this transfer to take place during sleep is that if identical neocortical networks are engaged in encoding, storage and retrieval of information (Damasio, 1989; Khader et al., 2005), these networks are deprived from afferent inputs during sleep so that no interference would occur between memories being consolidated and external information being continuously processed during wake (McClelland et al., 1995; Kali and Dayan, 2004). This mechanism has been associated with a specific sleep stage, i.e., slow wave sleep (SWS, Plihal and Born, 1999; Marshall et al., 2006; Gais et al., 2007; Rasch et al., 2007) and has been inferred for declarative memories.

To specify off-line learning effects of sleep, the declarative/nondeclarative dichotomy of memory has been used most extensively as a unifying framework, where declarative memory refers to verbalizible knowledge of facts (semantic) and events (episodic), and non-declarative memory refers to gains in perceptual and motor skills (procedural), benefits from statistic and associative regularities, priming, etc. (Squire, 1992; Seger, 1994). Both the declarative and non-declarative memories may profit from sleep (Plihal and Born, 1997, 1999; Gais et al., 2000; Fischer et al., 2002; Marshall et al., 2004, 2006; Walker and Stickgold, 2006) but not in all conditions and not consistently (Rasch et al., 2009; Robertson, 2009) and it is still debatable how and which aspects of memory are affected by sleep-specific stages and mechanisms (Walker and Stickgold, 2004; Diekelmann et al., 2009; Robertson, 2009).

Recently, awareness at learning before sleep has emerged as a critical factor for the sleep-related memory consolidation (Marshall and Born, 2007). This emphasized the explicit/implicit dichotomy of memory (Reber and Squire, 1994; Seger, 1994; Forkstam and Petersson, 2005; Reder et al., 2009) distinguishing memories that individuals are aware or unaware of acquiring and recollection 
(explicit vs. implicit). It was first stressed by Cohen et al. (2005) that a sleep-dependent advantage of procedural memories in the serial reaction time task (SRTT) only occurs if during pre-sleep training, subjects were made explicitly aware of the occurrence of a regular sequence. When, in contrast, pre-sleep training was conducted under implicit conditions, gains at retest were not larger after sleep than after wake (Cohen et al., 2005). Other studies have confirmed that the information that is consciously (explicitly) processed during encoding is preferentially supported by sleep (Kuriyama et al., 2004; Drosopoulos et al., 2005; Fischer et al., 2006). However, associative knowledge that is acquired implicitly before sleep also can be consolidated (Spencer et al., 2006) or transformed by sleep (Yordanova et al., 2008). It has been further proposed that it is the interaction between different types of memories, either at learning or during sleep, that affects the consolidation process (Born and Wagner, 2004; Fischer et al., 2006).

It is recognized, however, that to characterize explicit and implicit memories, it is necessary to address issues related to the nature of the acquired knowledge and its representation (explicit vs. implicit) and issues related to the nature of the acquisition and retrieval processes (explicit vs. implicit, controlled vs. automatic, intentional vs. incidental), and their functional interactions (Forkstam and Petersson, 2005). According to a recent model, explicit and implicit memory systems may not be postulated as independent systems based on consciousness but they may be separated by specifying both a representation and a process that operates on the representation (Reder et al., 2009). For the implicit memory system, distinct sub-systems have been previously suggested depending on the type of information being processed out of awareness (e.g., Willingham, 1997). For the working memory system that integrates consciously processed information at encoding (Baddeley and Hitch, 1974), two components also have been specified: 'the central executive' as a main component of 'fluid' (or processing-based) capacities such as attention, and 'the episodic buffer' as a main component of 'crystallized' (or knowledge-based) capacities (Baddeley, 2000). Furthermore, these two components are linked to distinct neural substrates, with the executive control being sub-served by prefrontal-parietal networks, and the episodic buffer being associated with the inferior parietal cortex (Rugg et al., 2008; Vilberg and Rugg, 2008). Given that the parietal cortical regions are elements of a hippocampal-cortical network sub-serving memory (Vincent et al., 2006), it is notable that the off-line memory consolidation at systems level mediated by sleep may involve differentially the systems activated at encoding (Gais et al., 2007). Thus, novel frameworks for off-line memory processing consider these knowledge-based and processing-based explicit/ implicit distinctions and give support to new detailed classifications of memory (Robertson, 2009).

To shed further light on the role of sleep for off-line learning, the present study accounts for an extended classification of dynamic memory. Specifically, we propose that the distinction between 'explicit' and 'implicit' memories may be meaningfully made on two dimensions. The knowledge-based dimension refers to types of information: Explicit knowledge (ExK) denotes consciously perceived task information; Implicit knowledge (ImK) denotes task information that remains out of awareness. The processing-based dimension of the explicit-implicit distinction refers to modes of processing:
Explicit processing (ExP) includes perceptual, cognitive, and motor processes such as stimulus selection and search, attention focusing and maintenance, memorization, computation, decision making, response selection and execution, etc., all of which are consciously guided by executive control systems. In contrast, implicit processing (ImP) involves mechanisms such as perceptual and motor proceduralization, statistical and associative learning, etc. Depending on the instructions these processes may occur without awareness and are not guided by executive control systems although they may modify behavioral responses. Each type of task information can be processed in two ways, explicitly and implicitly, and more importantly, these processes may occur in parallel.

Here, data from a previous study of Yordanova et al. (2008) were re-analyzed under the guideline of the outlined $2 \times 2$ scheme in order to specify the effects of different sleep stages, slow wave sleep (SWS) and rapid eye movement (REM) sleep, on the delineated functional aspects of off-line memory consolidation. The Number Reduction Task (NRT, Woltz et al., 1996; Frensch et al., 2002; Haider and Rose, 2007) provided a condition, in which the knowledge-based and processing-based dimensions of the explicitimplicit distinction could be dissociated. Similar to serial learning tasks, the NRT has two levels of organization, overt and covert. As detailed in Materials and Methods section and illustrated in Figure 1, each trial of the NRT consists of a string of several digits. At the overt level, subjects have to process the digits and produce consecutive responses following two operational rules. The covert level of NRT organization is that unmentioned to the subjects, all strings are generated according to an abstract regularity according to which the last three responses in a string always mirror symmetrically the preceding three responses, so that the second response in each trial is identical to the final solution (Figure 1). If capable of comprehending this regularity, participants could abruptly shortcut sequential responding. This regularity is abstract because the actual digit strings and responses change from trial to trial. The four cells of combinations given by the two dimensions of the explicit-implicit distinction can be defined in the NRT as follows (Figure 2).

\section{IMPLICIT PROCESSING OF EXPLICIT KNOWLEDGE (ImP-EXK)}

ImP-ExK refers to the procedural gain in instructed performance skills. Automation, proceduralization or operationalization occur implicitly and are generally reflected by behavioral improvement (decrease in response speed and error rate). As detailed below, several aspects of procedural learning can be distinguished in the NRT (perceptual, cognitive, or motor) by separate analysis of specific responses within a trial.

\section{EXPLICIT PROCESSING OF EXPLICIT KNOWLEDGE (EXP-EXK)}

ExP-ExK refers to the conscious controlled application of the instructed rules for NRT performance. As specified in the Materials and Methods section, there were two rules for stimulus-response processing in the NRT, the 'identity' and the 'difference' rule. Being computationally simpler than the 'difference' rule, the 'identity' rule would produce shorter response times (RT) than the 'difference' rule when these rules are deliberately applied. Thus, the RT difference between responses governed by the two instructed rules provides an estimate for the ExP-ExK processing. 


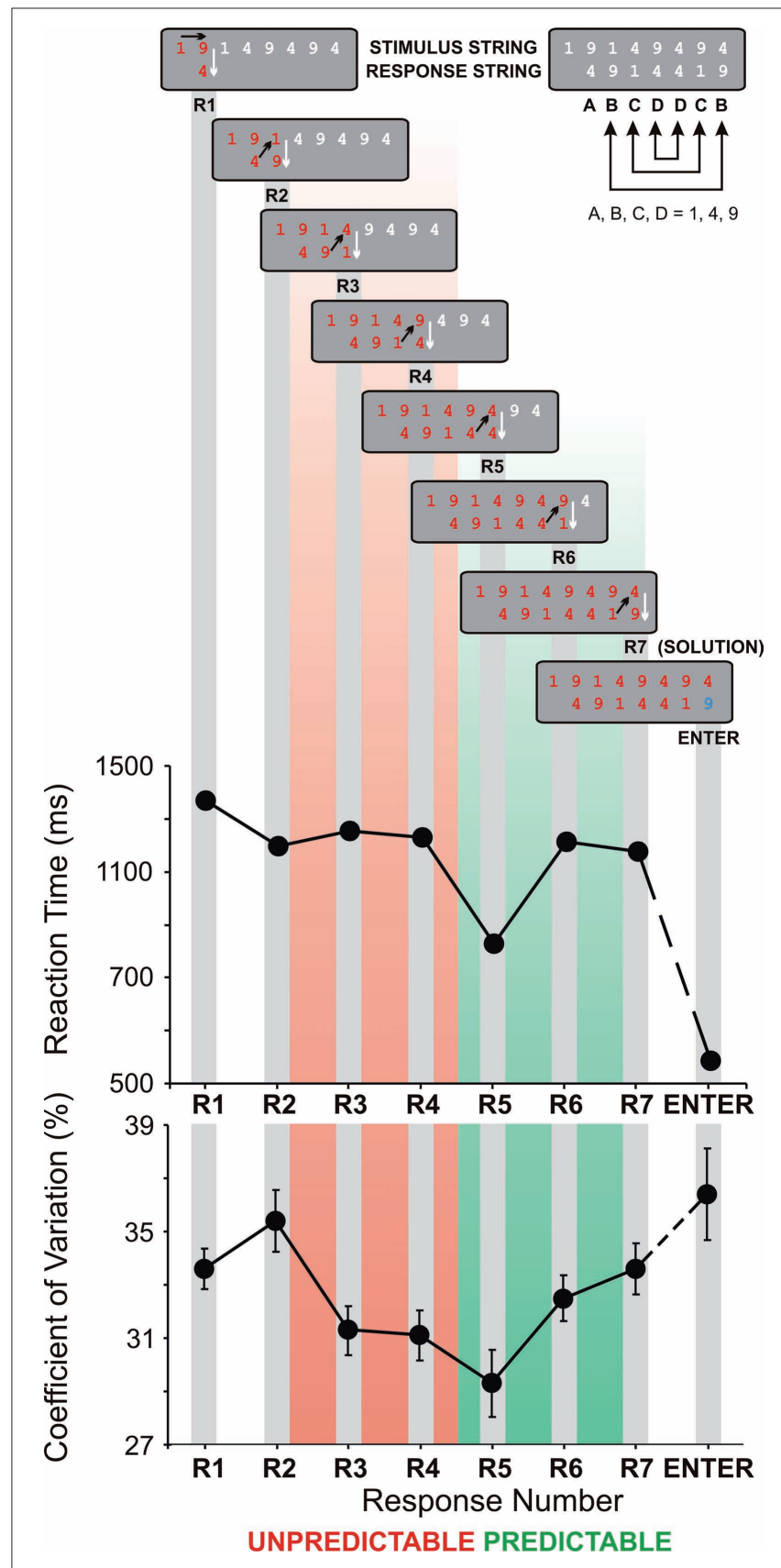

FIGURE 1 | Schematic presentation of the Number Reduction Task (NRT). A single representative string is shown. Black arrows present the consecutive steps in NRT task performance (e.g., the first two numbers 1 and 9 in the stimulus string lead to response $4(\mathrm{R} 1)$, then the same response (4) is compared with the next number from the stimulus string (1) leading to response 9 (R2), and so on). On the right, the structure of responses is given by letters showing the mirror structure of the response string (BCD-DCB). The final result is the last response (R7) marked with SOLUTION which has to be confirmed by pressing ENTER. At the bottom, mean reaction times and their coefficients of variance from all conditions and groups are shown for different response numbers ( $\mathrm{R} 1$ to $\mathrm{R} 7$ ). Standard error bars in the coefficient of variance give supportive information about the stability of performance. Additionally, according to their predictability during NRT processing, responses are divided into two response types: $\mathrm{R} 2$ to $\mathrm{R} 4$ - unpredictable (marked in red), and R5 to R7 - predictable (marked in green).

\section{IMPLICIT PROCESSING OF IMPLICIT KNOWLEDGE (ImP-ImK)}

ImP-ImK refers to the acquirement of implicit knowledge about the hidden regularity of the NRT structure. This implicit learning has been verified by the speeding of responses that can be predicted relative to responses that cannot be predicted by that regularity (Frensch et al., 2002; Lang et al., 2006; Haider and Rose, 2007; Yordanova et al., 2008). It has been demonstrated that RT decrease to predictable responses is not due to a fast short-term proceduralization within the string because when the same strings are processed without the mirror regularity being introduced, responses at last positions in the string are not faster (Rose et al., 2005). Therefore, RT decrease to predictable responses that is greater than RT decrease to unpredictable responses, is a reliable quantifier of ImP-ImK learning in the NRT (Yordanova et al., 2008).

\section{EXPLICIT PROCESSING OF IMPLICIT KNOWLEDGE (EXP-ImK)}

ExP-ImK refers to the generation of explicit knowledge (awareness) about the hidden regularity of the NRT. The culmination of that process is the insight, which is marked typically as a sudden awareness of that unobvious regularity (Frensch et al., 2002; Rose et al., 2002). Importantly, activation of explicit mechanisms for processing of covert NRT information can be assessed even before insight, and also in all subjects, irrespective of whether they subsequently will or will not comprehend the hidden regularity. One such marker is an increase in performance variability (Frensch et al., 2002; Rose et al., 2002; Lang et al., 2006; Yordanova et al., 2009). Even in implicit learning conditions, controlled processing may lead to conscious search, task exploration and hypotheses testing, which destabilizes ongoing performance (Frensch et al., 2002; Lang et al., 2006). Therefore, performance variability would reflect the extent to which covert information is actively searched for by the controlled processing systems.

To summarize, the present study analyzed the effects of specific sleep stages on the consolidation of different types of functional knowledge, explicit and implicit, processed either explicitly or implicitly. Relevant parameters of four different knowledge conditions $(2 \times 2$ scheme: ImP-ExK, ExP-ExK, ImP-ImK, ExP-ImK) of NRT performance were measured before and after sleep. One objective was to specify the functional domain of sleep-related memory consolidation by identifying both knowledge and processing mechanisms as explicit and implicit. A second objective was to shed light on the effects of different sleep stages, SWS and REM sleep, on different types of memories and their interactions (Born and Wagner, 2004). It was further aimed to test if sleep-dependent benefits of any particular type of functional knowledge may be specifically associated with subsequent post-sleep insight to the NRT regularity (Wagner et al., 2004; Yordanova et al., 2008). Although off-line learning of the NRT also takes place during wake (Wagner et al., 2004), here only the contribution of different sleep stages to these processes were addressed with regard to a detailed classification of dynamic memory.

\section{MATERIALS AND METHODS SUBJECTS}

For the current study, data from Yordanova et al. (2008) were reanalyzed. A total of 68 healthy students (18-28 year old) without any history of sleep disturbances or psychiatric or neurological 


\section{KNOWLEDGE}

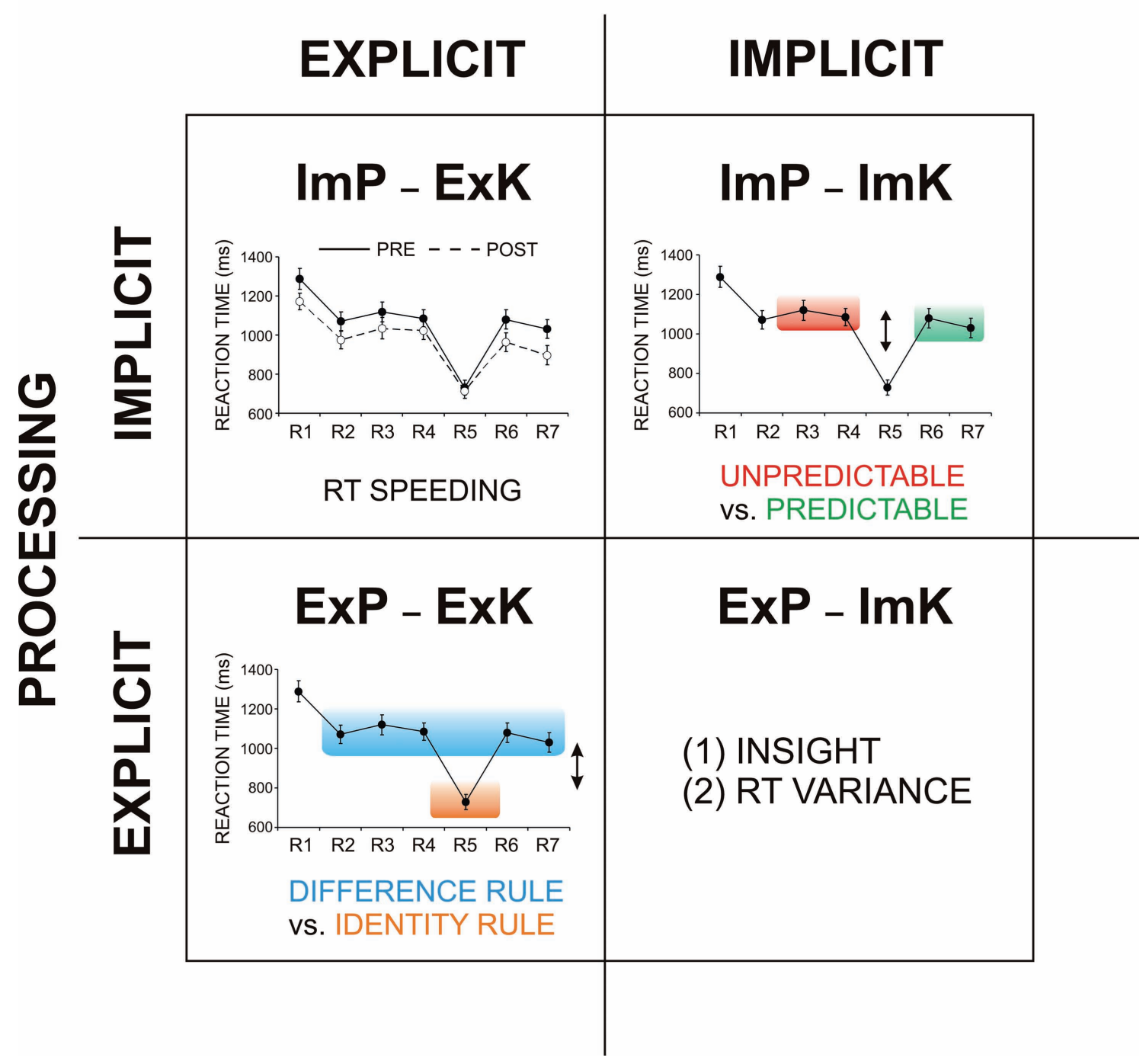

FIGURE 2 |Two types of processing (explicit, implicit) vs. two types of knowledge (explicit, implicit) and their measures. ImP-ExK (implicit processing of explicit knowledge measured by RT speeding), ExP-ExK (explicit processing of explicit knowledge measured by RT difference related to the conscious application of two rules, the 'identity' rule and the 'difference' rule, for NRT performance), ImP-ImK (implicit processing of implicit knowledge measured by RT difference for the unpredictable and predictable response types), ExP-ImK (explicit processing of implicit knowledge measured by the number of subjects gaining insight and by the RT variance). R1 to R7 response numbers. disorders had participated in that study. All subjects spent an adaptation night in the sleep laboratory including placement of electrodes. Subjects were paid for their participation and gave informed written consent prior to the study, which was approved by the local ethics committee.

Participants who gained explicit knowledge already at initial practice (indicated by short-cut responding in the NRT) were excluded from further analyses (four subjects in the early-night group and five subjects in the late-night group). One more subject was excluded from the early-night group because of staying awake $40 \%$ of the time, and one subject from the early-night group and two more subjects from the late-night group were excluded because of low quality of sleep EEG. Thus, 29 subjects ( 9 females) from the early-night group and 26 subjects ( 12 females) from the late-night group were used for statistical comparisons.

\section{TASK}

The task used by Yordanova et al. (2008) is illustrated in Figure 1 by an example trial. On each trial, a different string of eight digits was presented. Each string was composed of the digits 1, 4, and 9. For each string, subjects had to determine a digit defined as the final result (solution) of the task trial. This could be achieved by sequentially processing pairs of digits from left to right according to two simple rules: (1) The 'identity rule' states that the result of 
two identical digits is the same digit (e.g., 4 and 4 gives 4, Figure 1 - R5). (2) The 'difference rule' states that the result of two nonidentical digits is the remaining third digit (e.g., 1 and 9 gives 4, Figure 1 - R1). The 1, 2, and 3 keys on the PC numeric pad were labeled accordingly 1, 4, and 9 and served as response keys. The entered responses appeared on the screen and remained there until the end of the trial, thereby forming a response sequence below the stimulus sequence.

To produce the first response, comparisons are made between the first and the second digits from the stimulus string (Figure 1 $-\mathrm{R} 1)$. After processing the first two digits, comparisons are made between this result (appearing in the line below the stimulus string) and the next digit from the stimulus string, then between the response digit of this new processing and the next stimulus string digit, and so on. Thus, applying the two rules, subjects generate a string of seven responses, with the last one indicating the final result (solution) to be confirmed by pressing the 'Enter' key on the numeric pad. The time for any single response was limited to $4 \mathrm{~s}$ and to a total of $12 \mathrm{~s}$ for all responses until pressing 'Enter'. Pressing the 'Enter' key was followed by a change of color of the entered final response on the screen, from red to blue. After another 1 -s period, feedback was provided. In case of a correct final result, all digits on the screen, in addition to the final one, changed their color to blue, whereas the German word for 'Wrong' appeared on the screen in case of an incorrect final result. The screen was cleared after another $0.5 \mathrm{~s}$, and the next trial started. Instructions stated that only the final result was to be determined for each trial and this could be done at any time. Importantly, unmentioned to subjects, all strings were generated according to the same underlying regularity which, if discerned, allowed an early determination of the final result. Specifically, all response sequences had the form $\mathrm{ABCDDCB}$ (with $\mathrm{A}, \mathrm{B}, \mathrm{C}$, and $\mathrm{D}$ representing one of the digits 1 , 4 , or 9 ), i.e., the last three responses always mirrored the preceding three responses, so that the second response in each trial coincided with the final result (Figure 1). Thus, when gaining insight into this regularity, participants abruptly cut short sequential responding by pressing the 'Enter' key already after the second response (R2), whereupon the trial was finished and the next trial started. Note that this regularity is abstract because the actual digit strings and responses changed from trial to trial. Thus, discovery of the rule cannot simply be based on repetition of the same finger movements in all trials.

Reaction times (RTs) were measured continuously during task performance, separately for each response in the response string. RT of the first response (R1) was measured as the time from string appearance to the first key press. The RTs of the other responses (R2, R3, R4, R5, R6, R7, Enter) were measured as the time between the previous and the current key press.

\section{EXPERIMENTAL PROCEDURE}

Subjects were tested individually in a sound-attenuated room. Subjects performed a pre-sleep session of initial practice comprising of 3 task blocks and a post-sleep retest session of 10 task blocks, with 30 trials in each block. Only three blocks were used in the pre-sleep session to enable the initial formation of task representations, and much more (10) blocks were used in the post-sleep session to enable a reliable detection of insight, since in solvers, explicit knowledge generation in this version of the NRT emerges within 10 experimental blocks (Haider and Rose, 2007). Insight was automatically identified by the program when at least 24 correct short-cuts within the same block occurred, in which case the task was terminated. Initial practice was preceded by extensive standardized instructions given by the computer, which included a short practice block of 10 task trials. To assure correct understanding of the 'identity' and 'difference' rule, the practice block was repeated as long as the subject did not perform 10 trials without mistake. To investigate the effects of different sleep phases, the interval between initial practice and retest was filled with $3 \mathrm{~h}$ of sleep either in the early night, rich in SWS, or in the late night, rich in REM sleep.

In the early-night group (Early-NG), subjects came to the laboratory at about 21:00 h. After placement of electrodes (for EEG and standard polysomnographic recordings), they performed the three blocks of initial practice (including preceding computer-guided instructions) at about 22:00 $\mathrm{h}$ and thereafter went to bed at about 23:00 h. After $3 \mathrm{~h}$ of sleep in the early night they were awakened to perform the 10 blocks of NRT retesting. Subjects in the late-night group (Late-NG) reported to the laboratory at about 22:00 $\mathrm{h}$ and, after placement of electrodes, first slept for $3 \mathrm{~h}$ in the early night (to 'consume' SWS) before performing the initial practice at about $2: 30 \mathrm{~h}$. Then, they slept again for another $3 \mathrm{~h}$ in the late night (about $3: 30 \mathrm{~h}-6: 30 \mathrm{~h}$ ), followed by retesting in the morning. Subjects were only awakened from light sleep stages 1 or 2 to avoid cognitive disturbances that can occur after awakenings from SWS or REM sleep. As an additional control, subjective levels of sleepiness, activation, boredom, concentration, and motivation were assessed on 5 -point scales immediately before and after each session of initial practice and retest.

After the NRT retesting session, subjects filled in a questionnaire to probe their explicit knowledge related to the hidden task structure (beginning with open questions, followed by closed questions) as well as possible strategies used during task performance. An additional behavioral test comprised a speeded task in which 16 different strings were presented and subjects had to indicate the final result to each string within $2 \mathrm{~s}$ after string presentation. This test was performed immediately before administration of the questionnaire.

\section{ASSESSMENT OF EXPLICIT AND IMPLICIT KNOWLEDGE OF THE HIDDEN REGULARITY}

Presence of explicit knowledge after sleep was determined from NRT task performance and answers in the post-experimental questionnaire. Questionnaire data confirmed that all subjects whose insight into the hidden structure had been identified automatically by the task program due to the short-cut in sequential responding in the NRT were also able to verbalize the critical explicit rule knowledge correctly in open questions (i.e., using their own words), and they were also able to give correct solutions to new digit strings within $2 \mathrm{~s}$. Additional participants (three from the Early-NG and one from the Late-NG) were able to exactly verbalize the hidden mirror structure of the response strings in the questionnaire, and were therefore, also treated as ExK subjects although they had not put their explicit knowledge into action. Some questionnaire answers showed that the underlying common structure of all task trials also allowed detecting other short-cut rules than described 
in 2.2. These alternative ways of gaining insight into the hidden structure, likewise always associated with the immediate change to short-cut responding in the NRT, were considered as equivalent ways of acquiring explicit knowledge.

\section{SLEEP RECORDING AND ANALYSIS}

Sleep was recorded polysomnographically, including EEG recordings from left and right central sites (C3, C4), horizontal and vertical EOG, and EMG from chin electrodes. Sleep stages S1, S2, S3, S4, and REM sleep were classified in 30-s epochs according to Rechtschaffen and Kales (1968). SWS was calculated as the sum of time spent in sleep stages S3 and S4. Sleep parameters were compared by one-way ANOVA between early- and late-night sleep.

\section{PERFORMANCE ANALYSIS}

As reported in Yordanova et al. (2008), to evaluate differences between early and late-night sleep in the development of knowledge about the hidden regularity, $\chi^{2}$ tests were performed. For subjective ratings of sleepiness, activation, boredom, concentration, and motivation, $2 \times 2 \times 2$ ANOVAs were performed with the between-subjects factor Sleep Group (Early-NG vs. Late-NG) and the within-subjects factors Session (pre-sleep vs. post-sleep) and Time within session (beginning vs. end of session).

The following measures of RTs were newly evaluated in the present re-analysis, always taken as averages across trials from the last (third) block of the pre-sleep session and from the first block of the post-sleep session. Only the first block of the post-sleep session was used for two reasons: (1) to avoid any practice effects and assess task performance as a direct consequence of the preceding sleep, and (2) in this first post-sleep block, none of the subjects was aware of the hidden task regularity and the principles of NRT performance were as in the pre-sleep session. These measures are illustrated in Figure 2. RTs were analyzed both as original values and in normalized form, i.e., with RTs expressed as percentage of some specific response which was set to $100 \%$. This response was either the one with the maximum RT, in which case the one with the minimum RT was set to $0 \%$, thus producing a min-max normalization, or a specific pre-defined response, as will be detailed. Parameters were subjected to repeated-measures ANOVA with two between-subjects factors, Sleep Group (Early-NG vs. Late-NG) and Performance Group (Solvers vs. Non-solvers), and two within-subjects variables, Session (pre-sleep vs. post-sleep) and Response (R, depending on the specific type of knowledge tested, difference-rule related vs. identity-rule related; unpredictable vs. predictable, or R1 to R7). In separate analyses, relevant parameters were evaluated only for the pre-sleep session, with Sleep Group and Performance Group again included as between-subjects variables, and Response included as a within-subjects variable. When the Response factor had more than two levels, the Greenhouse-Geisser correction procedure was employed, with original degrees of freedom and corrected probabilities ( $\mathrm{p}$ ) being reported.

\section{Implicit Processing of Explicit Knowledge}

ImP-ExK refers to the procedural gain in performance skills. In the NRT, RTs are integrative outcomes of perceptual-motor and computational processes since pairs of digits are visually selected from the strings, are compared to one another in order to find a response which is then translated to pressing of the appropriate key. In contrast, the Enter response, with which the final response is confirmed, is free of this processing and virtually may represent a simple movement speed. Furthermore, in this version of the NRT, the $\mathrm{R} 5$ response is always associated with the identity rule requiring minimal computational operations. Thus, changes in RT to the final Enter-press would be able to capture with greatest reliability motor procedural learning, whereas changes in the RT to R5 would be able to isolate primarily perceptual-motor learning. Given that the Enter-response reflects individual differences in motor speed, ImP-ExK can be evaluated with finer precision when individual motor speed is subtracted from the integrative RT to R1-R7 (or when RTs to R1-R7 are normalized according to the Enter-RT). In this way, sleep-dependent implicit learning of explicit information can be analyzed separately for (1) motor processes (as reflected by Enter-RT), (2) perceptual-motor processes (as reflected by RT to R5 normalized to Enter-RT) and (3) integrative computational processes (as reflected by RTs to R1 to R7 normalized to EnterRT and RT to R5). Normalized values (RTnorm) were calculated according to the corresponding base value RTb (RT-Enter or RTR5) following the equation:

RTnorm $=$ RTi/RTb

where RTi is the actual value. Original and normalized RTs were subjected to a Sleep Group (Early-NG vs. Late-NG) $\times$ Performance Group (Solvers vs. Non-solvers) $\times$ Session (pre-sleep vs. postsleep $) \times$ Response $(\mathrm{R} 1-\mathrm{R} 7$; Enter-RT) ANOVA with repeated measures.

\section{Explicit Processing of Explicit Knowledge}

ExP-ExK refers to the conscious controlled application of the two instructed rules. The identity rule is computationally simpler than the difference rule and is further facilitated by the response-repetition effect. The conscious application of these principles produces a significant difference in RTs. Figure $\mathbf{1}$ (bottom) illustrates RTs across groups and blocks in the pre-sleep session and verifies the ExP-ExK effect. As mentioned above, R5 always followed the identity rule, whereas responses R2, R3, R4, R6, R7 always followed the difference rule. $\mathrm{R} 1$ was associated with both rules $(30 \%$ of the trials followed the identity rule, and $70 \%$ followed the difference rule). The ExP-ExK effect is demonstrated by the significantly shorter RTs to R5 relative to the difference-rule responses ( $\mathrm{R}$ effect, $F(6 / 306)=47.9, p<0.0001$; for R5 simple contrasts $(F(1 / 51)=59.8-169.2, p<0.001)$, as well as by the significant difference between RT to identity-rule-related and difference-rulerelated R1 responses $(F(1 / 51)=27.4, p<0.001)$. Additionally, Figure 1 shows that Enter-RT was substantially shorter than any of the responses in the string ( $\mathrm{R}$ effect, $F(7 / 357)=152.7$, $p<0.0001$; simple contrasts for Enter-RT, $F(1 / 51)=138.0-538.4$, $p<0.00001)$ verifying that it virtually represents simple movement speed.

The significance of these effects indicates that changes of the RT difference between R5 and difference-rule responses would index a change in the mode of conscious processing strategy. Here, to measure these relations across sleep, RTs were min/max normalized within the pre-sleep and post-sleep blocks. This normaliza- 
tion assigns a value of 1 to the maximal RT in a block and a value of 0 to the minimal RT in a block, with other RTs being accordingly re-computed. In this way, the distances among RT to different responses are emphasized, with confounding from absolute response speed being minimized.

For analysis of ExP-ExK, original and normalized RTs were subjected to a Sleep Group $($ Early-NG vs. Late-NG) $\times$ Performance Group (Solvers vs. Non-solvers) $\times$ Session (pre-sleep vs. postsleep) $\times$ Response (R2, R3, R4, R5, R6, R7) ANOVA. To evaluate the relations between identity rule-related $\mathrm{R} 5$ response and difference rule-related responses R2, R3, R4, R6 and R7, a min/max normalization was applied separately for the last pre-sleep block and the first post-sleep block. Normalized values (RTnorm) were calculated for each individual according to the equation:

RTnorm $=$ RTi $/($ RTmax - RTmin $)$

where RTi is the actual RT value, RTmax is the maximal individual average RT value within a block, and RTmin is the minimal individual average $\mathrm{RT}$ value within a block.

\section{Implicit Processing of Implicit Knowledge}

ImP-ImK refers to the acquirement of implicit knowledge about the covert regularity of the NRT structure. A reliable quantifier of ImPImK learning in the NRT is obtained when RT decreases between pre-sleep and post-sleep session more for predictable responses than for unpredictable responses (Yordanova et al., 2008). R5 is not included in this analysis, being exclusively determined by the identity rule. To support a balanced design, R3 and R4 were analyzed as unpredictable responses, and R6 and R7 were analyzed as predictable responses.

Original and min/max normalized RTs were analyzed. The ANOVA design was Sleep Group $\times$ Performance Group $\times$ Session $\times$ Predictability (unpredictable vs. predictable).

\section{Explicit Processing of Implicit Knowledge}

ExP-ImK refers to the generation of explicit knowledge (awareness) about the hidden regularity of the NRT (i.e., to the acquisition of explicit knowledge about the predictability of responses R5, R6, R7). While insight is the culmination of that process, activation of explicit mechanisms for processing of covert NRT information can be assessed before insight, because even in implicit learning conditions, controlled processing may lead to conscious search, task exploration and hypotheses testing, which destabilizes ongoing performance. This is reflected by deviations from straightforward responding. For analysis of ExP-ImK, coefficients of variance (CV) of RT were computed according to the equation:

$$
\mathrm{CV}(\%)=\mathrm{SD} \times 100 / \mathrm{RTmean}
$$

where SD is the RT standard deviation and RTmean is the mean RT. Individual CV values were subjected to the Sleep Group $\times$ Performance Group $\times$ Session $\times$ Response ANOVA, with factor Response including the responses from R1 to R7.

A relevant constraint of this quantifier takes into account the fact that RT variability may increase as a function of attentional deficiency (trait or state dependent), which is accompanied by slower RTs, or higher impulsivity (trait and state dependent), which is accompanied by faster RTs. Therefore, performance variance can be accepted as a measure of ExP-ImK learning (1) when response speed remains within the normal range, and (2) when differences are response-specific rather than general (i.e., refer to particular, but not to all response types). In all analyses, the Greenhouse-Geisser correction procedure was applied to variables with more than two levels, with the original df being reported in the results.

Previous studies with the NRT have demonstrated that the transformation of implicit abstract knowledge to explicit is promoted by SWS in the early half of the night (Yordanova et al., 2008) and that implicit abstract task representations are reorganized primarily by the SWS (Yordanova et al., 2009). Also, SWS has been suggested to support declarative (explicit) memory system (Plihal and Born, 1997) or the interaction of implicit and explicit memory systems whereas REM sleep has been associated with implicit/procedural learning (Drosopoulos et al., 2005; Stickgold, 2005; Born et al., 2006; Fischer et al., 2006). Both knowledgebased and processing-based aspects could have contributed to these effects of sleep on memory consolidation, so that firm hypotheses cannot be made on the basis of previous results. Here, it was expected that dissociating the functional aspects of memory would help to further specify the role of sleep for memory consolidation mechanisms.

\section{RESULTS}

As detailed in Yordanova et al. (2008), sleep recordings confirmed the differential distribution of SWS vs. REM sleep. Subjects in the Early-NG had substantially more SWS than those in the Late-NG $(p<0.001)$, and subjects in the Late-NG, conversely, had substantially more REM sleep than those in the Early-NG $(p<0.001)$. The two groups did not differ in the proportions of other sleep stages $(p>0.2)$.

Ratings of subjective feelings of sleepiness, activation, tension, boredom, motivation, and concentration were obtained before and after each session of initial practice and retest. As reported in Yordanova et al. (2008), the two experimental groups did not differ on the whole in these variables, as indicated by non-significant main effects of early vs. late night (all $p>0.2$ ). However, subjects felt more sleepy and less activated, motivated and concentrated in task sessions performed in the middle of the night (i.e., initial training for late-night group, retest for early-night group) than in sessions performed in the evening (initial training for early-night group) or in the morning (retest for late-night group) ( $p<0.05$, for respective night-half $\times$ session interactions). A much stronger effect independent of sleep was an activating effect of task performance itself, i.e., subjects felt less sleepy and more activated at the end as compared to the beginning of a task session $(p<0.001)$.

Nine out of 29 subjects (31.04\%) from the Early-NG and 5 out of 26 subjects (19.2\%) from the Late-NG group could discover the hidden task regularity after sleep, with the rate of post-sleep solvers not differing between the two sleep groups $\left(\chi^{2}(1, n=55)=1.01\right.$, $p=0.32)$. Importantly, none of the solvers gained explicit knowledge of the NRT hidden structure before the forth (from 10) block of the post-sleep session. Therefore, in the first block after sleep 
analyzed here, all subjects were unaware of task regularity and processed the task as in the pre-sleep session by following the instructed rules ('identity' rule and 'difference' rule).

Because of the differences in subjective feelings before sleep, to rule out that any changes across sleep could be simply attributable to differences in the mode of processing that were already present before the critical post-learning sleep period, a comparison was performed between the two groups only for the pre-sleep session. Relevant parameters (original and normalized RTs) before sleep were subjected to a Sleep Group $\times$ Performance Group analysis. Although solvers and non-solvers were separated only on the basis of their post-sleep performance (gain of insight about NRT regularity) that occurred later than the first post-sleep block, the Performance Group was included in this first analysis of the pre-sleep session to see if individual differences in the mode of task information encoding that might have contributed to the gain of explicit knowledge after sleep in solvers, could have affected the initial pre-sleep learning. As a second step, changes were evaluated between the two sessions, from before sleep to after sleep.

\section{IMPLICIT PROCESSING OF EXPLICIT KNOWLEDGE Pre-sleep analysis}

Before sleep the effect of Sleep Group on RTs to R1-R7 and Enter responses was not significant $(F(1 / 51)=1.9, p=0.2)$, which was valid for each response type $(F(1 / 51)=0.1-1.7, p=0.2-0.7)-$ Figures 3A,B. The same result was obtained with R1-R7 RTs normalized to the Enter-RT (Sleep Group: $(F(1 / 51)=0.1, p=0.8$; for each response type $(F(1 / 51)=0.002-0.5, p=0.6-0.93)$ - Figure 3C. Main and interactive effects of Performance Group also were nonsignificant $(F(1 / 51)=0.002-2.6, p=0.1-0.9)$.

\section{Across sleep analysis}

Figure 3A demonstrates that RTs generally decreased after sleep (Session, $F(1 / 51)=29.6, p<0.001)$. As confirmed by the interaction of Session $\times$ Response $(F(6 / 306)=4.25, p=0.002)$, in contrast to responses R1, R2, R3, R4, R6 and R7 $(F(1 / 51)=8.6-57.8$, $p<0.005-0.0001$ for effects of Session on each response), RT to R5 only marginally changed across sessions $(F(1 / 51)=3.6, p=0.06$, Figures $3 \mathrm{~A}, \mathrm{~B})$, and RT to the Enter press did not change at all (Session, $F(1 / 51)=0.9, p>0.3)$. There were no overall differences between Sleep Groups (all $F<0.7, p>0.5$ ). RT to Enter tended to be longer in solvers than non-solvers only after sleep $(F(1 / 51)=4.29$, $p=0.06$; Session $\times$ Performance, $F(1 / 51)=5.8, p=0.02)$.

RTs normalized according to the Enter-RT revealed differential effects of early and late sleep, as displayed in Figure 3C. As indicated by the significant Sleep Group $\times$ Session interaction $(F(1 / 51)=4.1, p<0.05)$, Enter-normalized RTs were generally reduced after early sleep $(F(1 / 27)=12.9, p<0.001)$, but not after late sleep $(F(1 / 24)=0.47, p=0.5)$. This effect was less expressed for $\mathrm{R} 5$ relative to the other responses (Session $\times$ Response $\times$ Sleep Group $F(6,306)=3.1, p=0.01$; Session $\times$ Response in the EarlyNG, $F(6 / 162)=3.8, p=0.008$; effects of Session $F(1 / 27)=4.5$, $p=0.05$ for $\mathrm{R} 5$, and $F(1 / 27)=8.1-15.6, p=0.008-0.001$ for other responses), whereas no differences between responses were found in the Late-NG in their lack of differences between sessions: Session $\times$ Response $(F(6 / 144)=1.3, p=0.25)$. The greater
$\mathrm{RT}$ reduction after early sleep to computationally complex items (R2, R3, R4, R6, R7) relative to the simple R5 item was additionally confirmed by analysis of RTs normalized to R5 (Session $\times$ Sleep Group, $F(1,51)=3.9, p=0.05$; effect of Session separately in the Early-NG, $F(1 / 27)=4.2, p=0.05$, in the Late-NG $F(1 / 24)=0.05$, $p=0.8$ ).

\section{EXPLICIT PROCESSING OF EXPLICIT KNOWLEDGE Pre-sleep analysis}

In the pre-sleep session, responses linked to the identity rule (R5) were significantly shorter than the responses linked to the difference rule $(\mathrm{R} 2-\mathrm{R} 7)(F(5 / 255)=45.8, p<0.001, F(1 / 51)=59.8$ $94.5, p<0.0001$ for each simple R5 comparison) - Figure 3B, but there were no effects of Sleep Group (all $F<1.7$ ) or Performance Group (all $F<0.67$ ). These effects were confirmed with RTs normalized to RT-Enter and to $\mathrm{min} / \mathrm{max}$ values $(F(5 / 255)=49.3$, 58.9; $p<0.001, F(1 / 51)=72.3-125.7,110.1-340.6 ; p<0.0001$ for each simple R5 comparison). Again, main and interactive effects of Sleep Group and Performance Group were not significant (all $F<1.0$ ).

\section{Across sleep analysis}

Figures $3 \mathrm{~A}-\mathrm{C}$ shows that responses linked to the identity rule (R5) were overall substantially shorter than those linked to the difference rule (R2-R7), main effect of Response $F(5 / 255)=44.5$, $p=0.001, F(1 / 51)=59.8-138.5, p<0.0001$, for comparison of each response R2, R3, R4, R6, R7 to R5. Although this difference from R5 slightly decreased from the pre-sleep to the postsleep session (Session $\times$ Response, $F(5 / 255)=2.7, p=0.02$ ), it was well significant both in the session before sleep (as reported above) and in the session after sleep $(F(5 / 255)=45.2, p<0.001$; $F(1 / 51)=32.1-154.1, p<0.0001$ for each simple R5 comparison). There were no effects of Sleep Group (all $F<3.0$ ) nor of Performance Group (all $F<1.8$ ).

\section{IMPLICIT PROCESSING OF IMPLICIT KNOWLEDGE Pre-sleep analysis}

Before sleep RTs to predictable (R6, R7) responses were already faster than RTs to unpredictable (R3, R4) responses (Predictability, $(F(1 / 51)=18.1, p<0.001)$ but this effect was present independently of the sleep group (Predictability $\times$ Sleep Group, $(F(1 / 51)=1.7$, $p=0.2$ ) or performance group (Predictability $\times$ Performance Group $F(1 / 51)=0.9, p=0.4)$. Analyses with normalized RTs yielded the same result (normalized to RT-Enter, $\mathrm{min} / \mathrm{max}$ : Predictability, $(F(1 / 51)=17.7,14.2 ; p<0.001 ;$ Predictability $\times$ Sleep Group, $(F(1 / 51)=0.95,1.4 ; p>0.25$; Predictability $\times$ Performance Group $F(1 / 51)=0.84,1.3 ; p>0.25)$.

\section{Across sleep analysis}

RTs to unpredictable and predictable response types decreased in the post-sleep session $(F(1 / 51)=39.6, p<0.001$, Figure 3A). But this decrease was greater for predictable than unpredictable responses (Session $\times$ Predictability, $F(1 / 51)=4.27, p<0.05$ ), which was manifested in a greater difference between sessions for predictable responses (Figure 3D): Effect of Session on predictable 


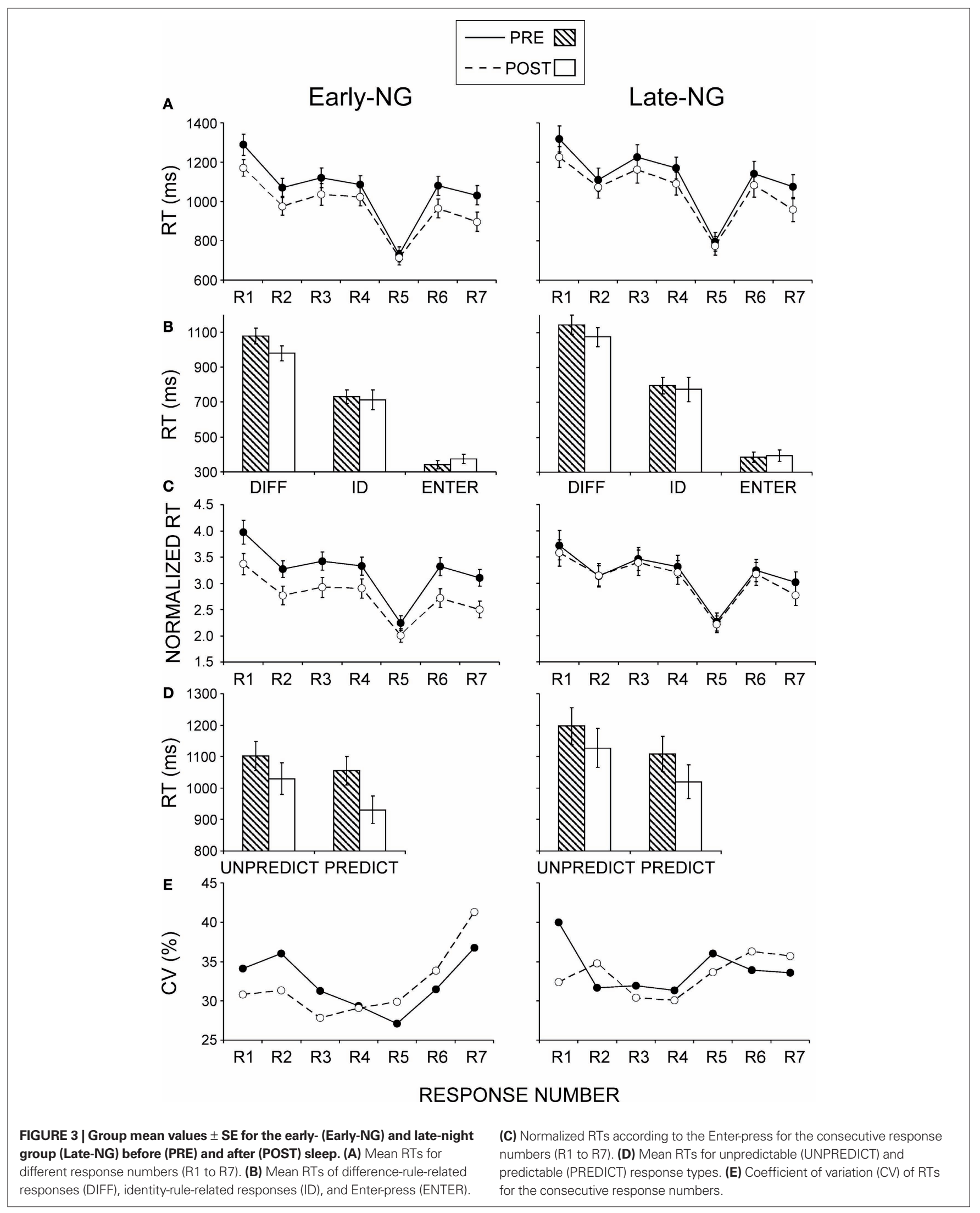


responses: $F(1,51)=47.6, p=0.0001$; on unpredictable responses: $F(1,51)=9.34, p=0.005$. There were no effects of Sleep Group (all $F<1.05$ ) nor of Performance Group (all $F<0.8$ ). These effects were replicated with normalized data.

\section{EXPLICIT PROCESSING OF IMPLICIT KNOWLEDGE \\ Pre-sleep analysis}

In the pre-sleep session, performance variance did not differ between the early- and late-night sleep groups (Sleep Group, $F(1 / 51)=0.7, p=0.4)$, nor between the groups of solvers and non-solvers (Performance Group, $F(1 / 51)=1.03, p=0.3$ ). Also, no interactions of between-group variables with response type were observed $(F<1.5, p>0.2)$.

\section{Across sleep analysis}

Response variance was larger for Solvers than Non-Solvers (Performance Group: $(F(1 / 51)=8.51, p=0.005$, Figure 3E). Importantly, as shown in Figure 4, this only applied to the post-sleep session (Session $\times$ Performance Group, $F(1 / 51)=8.67, p=0.005$; Performance Group after sleep $F(1 / 51)=10.03$, $p=0.003$; before sleep non-significant as reported above), and particularly to the predictable responses in the post-sleep session (Session $\times$ Response $\times$ Performance Group, $F(6 / 306)=2.5, p=0.03$; Session $\times$ Performance Group for R1 to R4, $F(1,51)<1.6, p>0.2$; in contrast for R6: $F(1,51)=6.42, p=0.014$; for R7: $F(1,51)=4.9, p=0.03)$.

According to the results, the effects of SWS and REM sleep on implicit processing of explicit information were different since an improvement in the post-sleep relative to the pre-sleep session was only found in the early-night group, in which SWS dominated. Pre-sleep analyses found no Sleep Group effects, which shows that group-specific processing or encoding of task information before sleep might not be the source of group differences after sleep. To provide further evidence that the post-sleep differences between the two sleep groups might not originate from the mode of encoding (Rugg et al., 2008), correlational analyses were performed for the gain in implicit processing of explicit information and the presleep parameters of the four processing modes. The gain in implicit processing of explicit information was computed as the post-sleep minus pre-sleep difference in RTs normalized to the RT-Enter. The pre-sleep parameters related to the four processing modes were: ImP-ExK - RT, RT normalized to Enter, RT normalized to R5; ExP-ExK - min/max normalized RT to identity rule and difference rule related responses; ImP-ImK - the pre-sleep difference between unpredictable and predictable responses in original and $\mathrm{min} / \mathrm{max}$ normalized RTs, and ExP-ImK - CV to different response types. None of these correlational analyses yielded significant results (absolute values of Pearson correlation coefficients, $r=0.03-2.5$, $p=0.1-0.9, n=55)$.

A further control analysis aimed at excluding the possibility that the differences in implicit processing of explicit information between the early- and late-night groups might simply reflect differences in on-line learning abilities between the groups (i.e., speed of performance improvement over time-on-task) that might have affected the results from the blocks included in the present analyses. To control for this effect, the ImP-ExK parameters were analyzed for the three pre-sleep blocks. If the early- and late-night groups originally differed in the speed of learning, this would be evinced already in the pre-sleep session by significant Sleep Group $\times$ Block interactions. For none of the analyses of RT, RT to the Enter press, and RT normalized to Enter-RT, was there a significant Sleep Group or Sleep Group $\times$ Block $(\times$ Response type $)$ effect $(F(1 / 53)=0.03-0.7$, $p=0.4-0.87 ; F(2 / 106)=0.13-0.57, p=0.53-0.85)-$ Figures 5A,B. Alternatively, if sleep stages in the early vs. late night modulated the differences in the ImP-ExK parameters, a significant Sleep Group effect would be expected for all blocks after sleep. To test this, data from the 10 post-sleep blocks were analyzed. Subjects who gained insight after sleep and short-cut responses were excluded from these analyses, since they did not have recordings from all 10 post-sleep blocks (see Materials and Methods, 'Assessment of Explicit and Implicit Knowledge of the Hidden Regularity'). Thus,

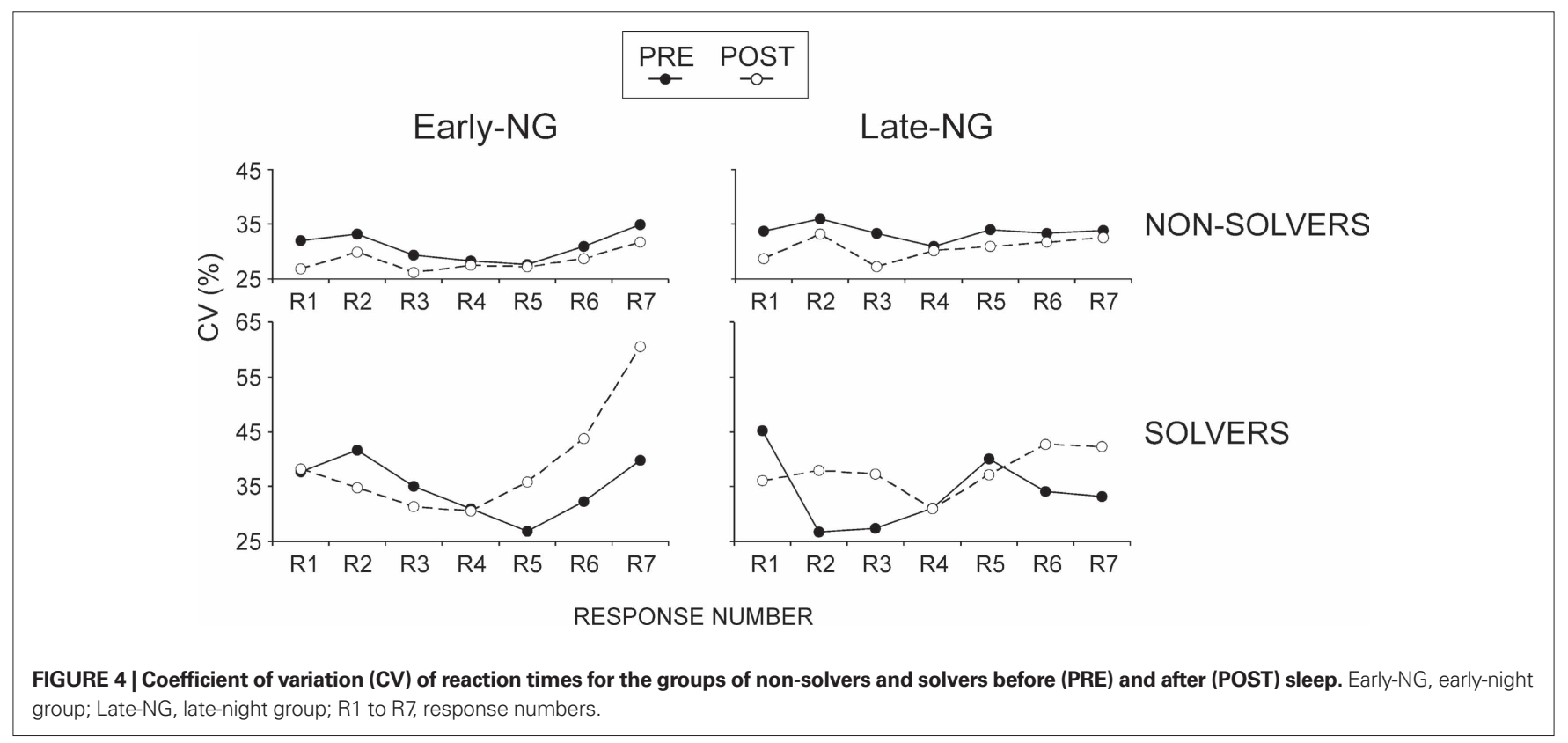


data of 23 subjects from the Early-NG and 22 subjects from the Late-NG were used. The main effect of Sleep Group was significant for the RT normalized to the Enter-RT $(F(1 / 44)=4.5, p=0.05)$, in contrast to RT and RT to the Enter press $(F(1 / 44)=0.067-0.62$, $p=0.4-0.8)-$ Figures 5A,B. No differences were found between the two sleep groups in the post-sleep dynamics of RT and RT to Enter $(F(9 / 396)=0.66-1.3, p=0.25-0.63)$ - Figure 5A, while the post-sleep difference in the RT normalized to Enter-RT even increased in the course of task performance $(F(9 / 396)=2.5$, $p=0.03)$ - Figure 5B.

\section{DISCUSSION}

The present study used the number reduction task to assess how two aspects of explicitness, explicitness of task information, and explicitness of task processing may affect off-line learning during sleep. (1) Based on availability to conscious awareness, the information of the task was divided into its explicit aspects (i.e., task information processed by applying the instructed rules which subjects were aware of: identity rule and difference rule) and its implicit aspects (i.e., task information of the hidden regularity which the subjects were not aware of: symmetric organization of responses). (2) Based on whether information was under the conscious control

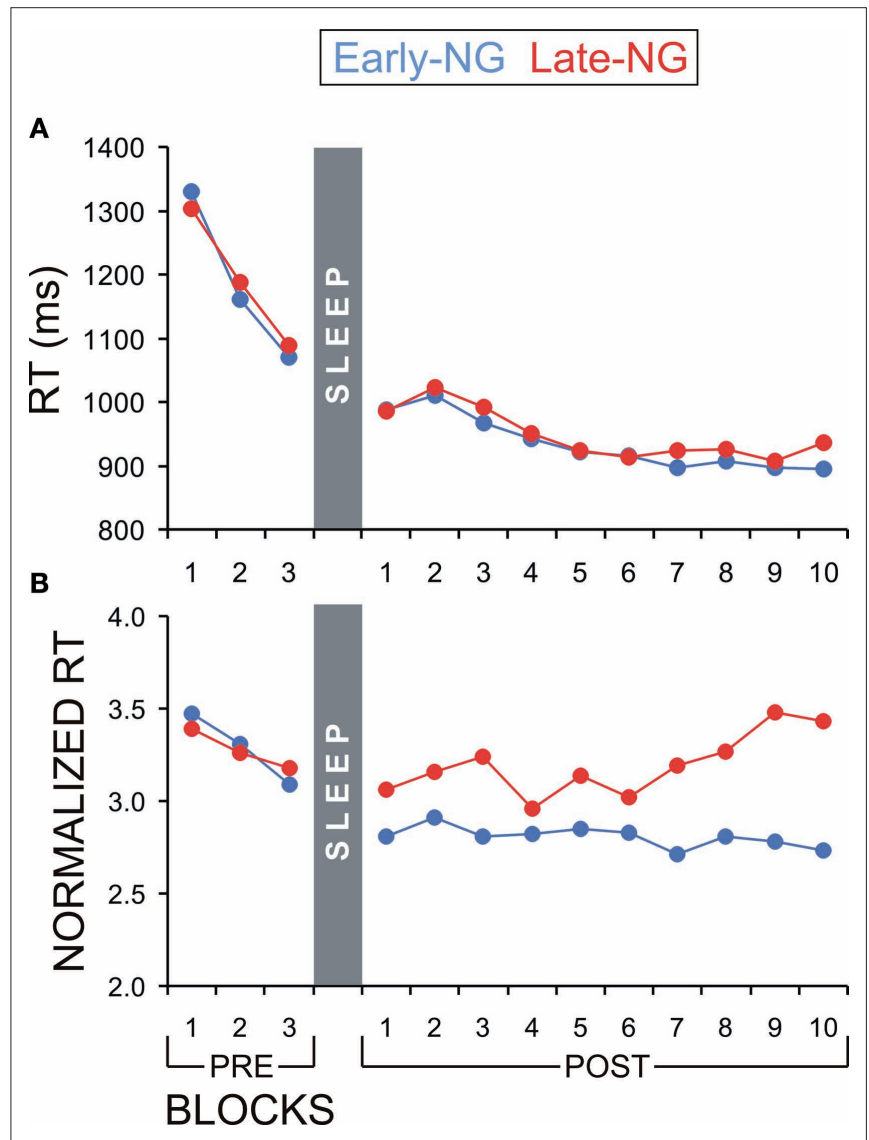

FIGURE 5 | Reaction time (RT) dynamics of NRT blocks before (PRE) and after (POST) sleep for the early- (Early-NG) and late-night group (LateNG). (A) Mean RT of all response types pooled together (R1 to R7). (B) Mean normalized RT according to the Enter-press of all response types pooled together (R1 to R7). of executive systems, processing was regarded as explicit or implicit. Either type of task information, explicit or implicit, could be processed in two ways, explicitly or implicitly. Introducing this distinction of awareness in two dimensions (knowledge-based vs. processing-based) allowed for evaluating the effects of different sleep stages, SWS and REM sleep, on specific functional aspects of memory consolidation.

One major result was that the effects of SWS and REM sleep could be separated for one of these functional aspects: Implicit processing of explicit information was improved in the post-sleep relative to the pre-sleep session only in the early-night group, in which SWS dominated. This was demonstrated by the postsleep gain in performance both for the simple computational operation needed to apply the identity-rule and, with an even greater gain in performance for the more complex computational operation needed to apply the difference-rule. These effects may be suspected to stem from the mere duration of the time interval between the pre-sleep and post-sleep sessions leading to consolidation (i.e., to be time-dependent, Robertson et al., 2004), or from the different times of the night when the sessions took place, rather than from the particular intervening sleep. Yet, the time intervals between pre- and post-sleep sessions were equally long in the two sleep groups, and the pre-sleep measures recorded in the evening for the early-night group, and at mid-night for the late-night group did not differ at all, thus indicating that these factors may not be critical determinants of the detected betweengroup differences. Results from correlational analyses also confirmed that the improvement of implicit processing of explicit information associated with the early-night sleep might not be produced by differences in pre-sleep encoding of task information. It may be also suspected that the post-sleep improvement in ImP-ExK parameters simply reflects an improvement in performance expected from preceding pre-sleep practice that would be observed in the absence of any off-line consolidation, the effects of which may be differentially modulated by the early- and latenight sleep. Although with the present experimental data it is not possible to address directly this issue, it may be argued that a preceding practice would affect similarly each of the response types. The results showed that the post-sleep speeding was not equal for different response types, being virtually absent for the Enter response type and being significant less expressed for the R5 (identity-rule-related) response type. Control analyses with all blocks further demonstrated a lack of between-group differences in on-line learning (time-on-task dynamics of performance) and a persistence of between-group differences for the whole post-sleep session. Although the present data do not permit firm conclusions about how exactly different sleep stages might have mediated effects expected from practice, they do show that the differential effects of early- and late-night sleep on implicit processing of explicit information can be attributed to a specific contribution of different sleep stages. It is left to demonstrate how similar intervening periods of wakefulness would affect the implicit processing of explicit information.

Importantly, neither early nor late sleep supported the speeding the Enter response, indicating that sleep did not promote offline procedural efficiency of simple movement. This result also shows that the post-sleep speeding of computational responses 
was probably not due to greater movement efficiency. Rather, the speeding of these responses reflects an improvement of computational skills, which can be explained with (a) faster sensorimotor integration, (b) learning of stimulus-stimulus associations, and (c) learning of stimulus-response associations. If sensorimotor integration alone was accelerated by early sleep, RT reductions would be similar for items with simple and complex computational requirements. The additional speeding for complex items therefore indicates that computational skills were facilitated perhaps on the basis of stabilized stimulus and response associations. Yet, this learning effect was only observed for task-instruction elements that were processed consciously in the pre-sleep session. Analysis of the electrophysiological activity recorded from the same data set (Yordanova et al., 2009) revealed that slow cortical potentials indicating the amount of controlled processing did not differ neither between unpredictable and predictable responses nor between early- and late-night sleep groups during implicit learning before sleep. These neurophysiologic observations provide evidence that encoding processes did not account for differences between sleep groups. Additionally, they demonstrated that only after early-night but not after late-night sleep was there a significant decrease in slow cortical potentials at occipito-parietal regions. Since the amount of controlled processing in task-specific cortical regions is reflected by the magnitude of slow cortical potentials at those regions, a facilitation of controlled visual processing was demonstrated (Yordanova et al., 2009). These observations of facilitated visual control provide neurophysiologic support to the notion that early sleep stabilizes stimulus and response associations. The important finding here is that only those associations among task elements that were processed consciously before sleep to fulfill instructed task goals appear to be strengthened by early night sleep. This result is consistent with previously reported effects of SWS on goal-based rather than on movement-based memories (Robertson, 2009), and additionally indicates that performance improvement of computational skills may not involve explicit processing mechanisms.

The implicit processing of the information having remained implicit (out of awareness) at pre-sleep encoding and being still out of awareness after sleep, was improved after both early and late sleep. Implicit off-line learning of covert task regularities has been reported previously for the serial reaction time task (Maquet, 2001; Peigneux et al., 2003). This effect, however, has been basically associated with the REM sleep dominating in the second half of the night, whereas according to the present observations, early- and late-night sleep did not differ in this respect. Our previous analysis of the same data set has demonstrated that SWS in the early night promotes the covert reorganization of implicit

\section{REFERENCES}

Baddeley, A. (2000). The episodic buffer: a new component of working memory? Trends Cogn. Sci. 4, 417-423.

Baddeley, A. D., and Hitch, G. J. (1974). Working memory. In The Psychology of Learning and Motivation, G. A. Bower, ed. (New York, Academic Press), pp. 47-89.
Born, J., Rasch, B., and Gais, S. (2006). Sleep to remember. Neuroscientist 12, 410-424.

Born, J., and Wagner, U. (2004). Awareness in memory: being explicit about the role of sleep. Trends Cogn. Sci. 8, 242-244.

Cohen, D. A., Pascual-Leone, A., Press, D. Z., and Robertson, E. M. (2005). Offline learning of motor skill memory: a

task representations (Yordanova et al., 2009) and facilitates the subsequent transition of implicit knowledge to explicit, whereas REM sleep in the late night preserves the implicit memories in their original form (Yordanova et al., 2008). Thus, different sleep stages may contribute, by means of different mechanisms, to the stronger consolidation of covert implicit information. However, since these effects were similar for the two sleep groups, it cannot be excluded that they were due to the time interval between the pre-sleep and post-sleep sessions (Cohen and Robertson, 2007; Mednick et al., 2009). To address this question, further studies with wake controls are required.

What definitely did not differ between sleep stages and did not benefit from either sleep stage was the explicit processing of either overt or covert information of the task. Although the lack of effect on explicit processing may reflect a covert compensation from increased cognitive efforts demonstrated previously by enhanced slow negative potentials (Yordanova et al., 2009), the relevant finding here is that the two sleep stages did not differ in this respect.

Notably, markers of conscious search (explicit processing) for covert regularities substantially increased in the post-sleep session in those individuals who subsequently gained explicit knowledge about the hidden task structure. This effect occurred equally for the early-night group and the late-night group. So, the effect might be independent of sleep although it cannot be excluded that the current statistical outcome may have been limited by the relatively small number of solvers. Nonetheless, given the existence of differences between solvers and non-solvers in each of the sleep groups, it may be speculated that sleep in general may lead to a strong activation of control processing systems to search for covert (implicit) information patterns.

In terms of interaction between explicit and implicit memory systems in the brain, the present results show that early- but not late-night sleep promotes the implicit access to explicit knowledge accumulated before sleep. Yordanova et al. (2008) have demonstrated that again early- but not late-night sleep promotes the explicit access to implicit knowledge acquired before sleep. Thus, an interaction between explicit and implicit memory systems appears only to occur in relation to early-night sleep, rich in slow wave sleep. A refined account of the explicit/implicit distinction of memory systems in terms of information and processes can contribute to clarify the role of sleep for off-line memory consolidation.

\section{ACKNOWLEDGMENT}

This work was supported by the Deutsche Forschungsgemeinschaft (SFB654/1-1).

double dissociation of goal and movement. Proc. Natl. Acad. Sci. U.S.A. 102 18237-18241.

Cohen, D.A., and Robertson, E. M. (2007). Motor sequence consolidation: constrained by critical time windows or competing components. Exp. Brain Res. 177, 440-446.

Damasio, A. R. (1989). Time-locked multiregional retroactivation: a systems-level proposal for the neural substrates of recall and recognition. Cognition 33, 25-62.

Diekelmann, S., and Born, J. (2007). One memory, two ways to consolidate? Nat. Neurosci. 10, 1085-1086.

Diekelmann, S., Wilhelm, I., and Born, J. (2009). The whats and whens of sleepdependent memory consolidation. Sleep Med. Rev. 13, 309-321. 
Drosopoulos, S., Wagner, U., and Born, J. (2005). Sleep enhances explicit recollection in recognition memory. Learn. Mem. 12, 44-51.

Fischer, S., Drosopoulos, S., Tsen, J., and Born, J. (2006). Implicit learning explicit knowing: a role for sleep in memory system interaction. J. Cogn. Neurosci. 18, 311-319.

Fischer, S., Hallschmid, M., Elsner, A. L., and Born, J. (2002). Sleep forms memory for finger skills. Proc. Natl. Acad. Sci. U.S.A. 99, 11987-11991.

Forkstam, C., and Petersson, K. M. (2005). Towards an explicit account of implicit learning. Curr. Opin. Neurol. 18, 435-441.

Frensch, P. A., Haider, H., Rünger, D., Neugebauer, U., Voigt, S., and Werg, J. (2002). Verbal report of incidentally experienced environmental regularity: the route from implicit learning to verbal expression of what has been learned. In Attention and Implicit Learning, L. Jimenez, ed. (New York, John Benjamins), pp. 335-366.

Gais, S., Albouy, G., Boly, M., Dang-Vu, T. T., Darsaud, A., Desseilles, M., Rauchs, G., Schabus, M., Sterpenich, V., Vandewalle, G., Maquet, P., and Peigneux, P. (2007). Sleep transforms the cerebral trace of declarative memories. Proc. Natl. Acad. Sci. U.S.A. 104, 18778-18783.

Gais, S., Plihal, W., Wagner, U., and Born, J. (2000). Early sleep triggers memory for early visual discrimination skills. Nat. Neurosci. 3, 1335-1339.

Haider, H., and Rose, M. (2007). How to investigate insight: a proposal. Methods 42, 49-57.

Kali, S., and Dayan, P. (2004). Off-line replay maintains declarative memories in a model of hippocampalneocortical interactions. Nat. Neurosci. 7, 286-294.

Karni, A., Tanne, D., Rubenstein, B. S., Askenasy, J. J., and Sagi, D. (1994). Dependence on REM sleep of overnight improvement of a perceptual skill. Science 265, 679-682.

Khader, P., Burke, M., Bien, S., Ranganath, C., and Rösler, F. (2005). Content-specific activation during associative long-term memory retrieval. Neuroimage 27, 805-816.

Kirov, R., Weiss, C., Siebner, H. R., Born, J., and Marshall, L. (2009). Slow oscillation electrical brain stimulation during waking promotes EEG theta activity and memory encoding. Proc. Natl. Acad. Sci. U.S.A. 106, 15460-15465.

Krakauer,J.W., and Shadmehr, R. (2006). Consolidation of motor memory. Trends Neurosci. 29, 58-64.

Kuriyama, K., Stickgold, R., and Walker, M. P. (2004).Sleep-dependent learning and motor-skill complexity. Learn. Mem. 11, 705-713.

Lang, S., Kanngieser, N., Jaskowski, P., Haider, H., Rose, M., and Verleger, R. (2006). Precursors of insight in eventrelated brain potentials. J. Cogn. Neurosci. 18, 1-15.

Maquet, P. (2001). The role of sleep in learning and memory. Science 294, 1048-1052.

Marshall, L., and Born, J. (2007). The contribution of sleep to hippocampusdependent memory consolidation. Trends Cogn. Sci. 11, 442-450.

Marshall, L., Helgadóttir, H., Mölle, M., and Born, J. (2006). Boosting slow oscillations during sleep potentiates memory. Nature 444, 610-613.

Marshall, L., Mölle, M., Hallschmid, M., and Born, J. (2004). Transcranial direct current stimulation during sleep improves declarative memory. J. Neurosci. 24, 9985-9992.

McClelland, J. L., McNaughton, B. L., and O'Reilly, R. C. (1995). Why there are complementary learning systems in the hippocampus and neocortex: insights from the successes and failures of connectionist models of learning and memory. Psychol. Rev. 102, 419-457.

Mednick, S. C., Makovski, T., Cai, D. J., and Jiang, Y.V. (2009). Sleep does not facilitate implicit memory in a visual search task. Vision Res. 49, 2557-2565.

Peigneux, P., Laureys, S., Fuchs, S., Destrebecqz, A., Collette, F., Delbeuck, X., Phillips, C., Aerts, J., Del Fiore, G., Degueldre, C., Luxen, A., Cleeremans, A., and Maquet, P. (2003). Learned material content and acquisition level modulate cerebral reactivation during posttraining rapideye-movements sleep. Neuroimage 20, 125-134.

Peigneux, P., Orban, P., Balteau, E., Degueldre, C., Luxen, A., Laureys, S., and Maquet, P. (2006). Offline persistence of memory-related cerebral activity during active wakefulness. PLoS Biol. 4, e100. doi: 10.1371/journal.pbio.0040100.

Plihal, W., and Born, J. (1997). Effects of early and late nocturnal sleep on declarative and procedural memory. J. Cogn. Neurosci. 9, 534-547.

Plihal, W., and Born, J. (1999). Effects of early and late nocturnal sleep on priming and spatial memory. Psychophysiology 36, 571-582.

Rasch, B., Büchel, C., Gais, S., and Born, J. (2007). Odor cues during slow-wave sleep prompt declarative memory consolidation. Science 315, 1426-1429.

Rasch, B., Pommer, J., Diekelmann, S., and Born, J. (2009). Pharmacological
REM sleep suppression paradoxically improves rather than impairs skill memory. Nat. Neurosci. 12, 396-397.

Reber, P. J., and Squire, L. R. (1994). Parallel brain systems for learning with and without awareness. Learn. Mem. 1, 217-229.

Rechtschaffen, A., and Kales, A. A. (1968). Manual of Standardized Terminology, Techniques and Scoring System for Sleep Stages of Human Subjects, No. 204. Bethesda, MD, National Institutes of Health.

Reder, L. M., Park, H., and Kieffaber, P. D. (2009). Memory systems do not divide on consciousness: reinterpreting memory in terms of activation and binding. Psychol. Bull. 135, 23-49.

Robertson, E. M. (2009). From creation to consolidation: a novel framework for memory processing. PLoS Biol. 7, e1000019. doi: 10.1371/journal. pbio. 1000019 .

Robertson, E. M., Pascual-Leone, A., and Press, D. Z. (2004). Awareness modifies the skill-learning benefits of sleep. Curr. Biol. 14, 208-212.

Robertson, E. M., Press, D.Z., and PascualLeone, A. (2005). Off-line learning and the primary motor cortex. J. Neurosci. 25, 6372-6378.

Rose, M., Haider, H., and Büchel, C. (2005). Unconscious detection of implicit expectancies. J. Cogn. Neurosci. 17, 918-927.

Rose, M., Haider, H., Weiller, C., and Büchel, C. (2002). The role of medial temporal lobe structures in implicit learning: an event-related fMRI study. Neuron 36, 1221-1231.

Rugg, M. D., Johnson, J. D., Park, H., and Uncapher, M. R. (2008). Encodingretrieval overlap in human episodic memory: a functional neuroimaging perspective. Prog. Brain Res. 169, 339-352.

Seger, C. A. (1994). Implicit learning. Psychol. Bull. 115, 163-196.

Spencer, R. M., Sunm, M., and Ivry, R. B. (2006). Sleep-dependent consolidation of contextual learning. Curr. Biol. 16, 1001-1005.

Squire, L. R. (1992). Memory and the hippocampus: a synthesis from findings with rats, monkeys, and humans. Psychol. Rev. 99, 195-231.

Stickgold, R. (2005). Sleep-dependent memory consolidation. Nature 437 , 1272-1278.

Vilberg, K. L., and Rugg, M. D. (2008) Memory retrieval and the parietal cortex: a review of evidence from a dualprocess perspective. Neuropsychologia 46, 1787-1799.

Vincent, J. L., Snyder, A. Z., Fox, M. D., Shannon, B. J., Andrews, J. R.
Raichle, M. E., and Buckner, R. L. (2006). Coherent spontaneous activity identifies a hippocampal-parietal memory network. J. Neurophysiol. 96, 3517-3531.

Wagner, U., Gais, S., Haider, H., Verleger, R., and Born, J. (2004). Sleep inspires insight. Nature 427, 352-355.

Walker, M. P., Brakefield, T., Morgan, A., Hobson, J.A., and Stickgold, R. (2002). Practice with sleep makes perfect: sleep-dependent motor skill learning. Neuron 35, 205-211.

Walker, M. P., and Stickgold, R. (2004). Sleep-dependent learning and memory consolidation. Neuron 44, 121-133.

Walker, M. P., and Stickgold, R. (2006). Sleep, memory, and plasticity. Annu. Rev. Psychol. 57, 139-166.

Willingham, D. B. (1997). Systems of memory in the human brain. Neuron $18,5-8$.

Woltz, D. J., Bell, B. G., Kyllonen, P. C., and Gardner, M. K. (1996). Memory for order of operations in the acquisition and transfer of sequential cognitive skills. J. Exp. Psychol. Learn. Mem. Cogn. 22, 438-457.

Yordanova, J., Kolev, V., Verleger, R., Bataghva, Z., Born, J., and Wagner, U. (2008). Different roles of early and late night sleep in making implicit knowledge explicit. Learn. Mem. 15, 508-515.

Yordanova, J., Kolev, V., Wagner, U., and Verleger, R. (2009). Covert reorganization of implicit task representations by slow wave sleep. PLoS ONE 4, e5675. doi: 10.1371/journal. pone. 0005675 .

Conflict of Interest Statement: The authors declare that the research was conducted in the absence of any commercial or financial relationships that could be construed as a potential conflict of interest.

Received: 28 April 2009; paper pending published: 25 May 2009; accepted: 12 October 2009; published online: 30 October 2009.

Citation: Yordanova J, Kolev $V$ and Verleger R (2009) Awareness of knowledge or awareness of processing? Implications for sleep-related memory consolidation. Front. Hum. Neurosci. 3:40. doi: 10.3389/neuro.09.040.2009

Copyright (c) 2009 Yordanova, Kolev and Verleger. This is an open-access article subject to an exclusive license agreement between the authors and the Frontiers Research Foundation, which permits unrestricted use, distribution, and reproduction in any medium, provided the original authors and source are credited. 